\title{
Trinucleotide (GAA)n repeat expansion in two families with Friedreich's ataxia with retained reflexes
}

\author{
Mark W Kellett, Nicholas A Fletcher, Nicholas Wood, T Peter Enevoldson
}

\begin{abstract}
In occasional families in whom cases of classic Friedreich's ataxia (FRDA) coexist with affected cases with retained reflexes, linkage analysis has shown that both map to the FRDA locus on chromosome 9q1321.1. A gene $X 25$ has been identified within the critical region of the FRDA locus, and an intronic expanded GAA trinucleotide repeat has been found in most cases of FRDA. We report two further FRDA families in whom some patients with classic FRDA were areflexic whereas others had brisk reflexes. Molecular genetic analysis disclosed an abnormal trinucleotide repeat expansion within intron 1 of the FRDA gene in both phenotypes.
\end{abstract}

(F Neurol Neurosurg Psychiatry 1997;63:780-783)

Keywords: Friedreich's ataxia; trinucleotide repeat; retained reflexes; cardiomyopathy

Friedreich's ataxia (FRDA) and early onset cerebellar ataxia with retained reflexes (EOCA) are both autosomal recessive spinocerebellar ataxias. Preservation of reflexes is the main diagnostic criterion distinguishing FRDA from EOCA..$^{1-3}$ Some patients have EOCA accompanied by other features suggestive of FRDA such as cardiomyopathy, and this may cause diagnostic uncertainty as to whether they have EOCA or atypical FRDA. Further evidence for a phenotype resembling EOCA sometimes being a manifestation of FRDA comes from rare families containing affected members with classic FRDA and others with preserved or exaggerated reflexes. ${ }^{45}$

In classic FRDA, the gene is on chromosome $9 q 13-21.1 .^{6}$ EOCA is genetically heterogeneous ${ }^{7}$ but some cases have a cardiomyopathy and linkage to the FRDA locus. ${ }^{8}$ In other $9 q$ linked families, members with classic FRDA have affected sibs with retained reflexes. ${ }^{89}$ This suggests that the FRDA and a subgroup of EOCA may be allelic disorders. Recently (GAA)n triplet repeat expansions within intron 1 of the gene X25 in the critical region of the FRDA locus have been associ- ated with FRDA. ${ }^{10}$ This gene encodes a 210 amino acid protein, frataxin, which is expressed in those parts of the CNS which degenerate in FRDA. Classic FRDA is homozygous for the GAA trinucleotide expansion in $92 \%-95 \%$ of patients; the remainder are compound heterozygotes with one expanded FRDA gene and a point mutation of the corresponding allele. ${ }^{10-13}$ Using the criteria proposed by Harding ${ }^{3}$ about one quarter of patients homozygous for the abnormal trinucleotide repeat have atypical presentations: ${ }^{11} \mathrm{a}$ few of these patients have the EOCA phenotype.

Here, we report two families in which the classic FRDA and EOCA phenotypes coexisted. In both families; molecular genetic analysis disclosed trinucleotide repeat expansions of the FRDA gene showing that both phenotypes were caused by the same mutation.

\section{Case reports}

FAMILY A

Patient 1

A 21 year old woman presented with three years of unsteadiness and mild right hand weakness. She was thin with bilateral pes cavus but no kyphoscoliosis. She had a soft aortic systolic murmur. Fundoscopy was normal. Saccadic eye movements were normal and there was no nystagmus but visual pursuit movements were broken. She had a brisk jaw jerk, slow tongue movements, and a mild dysarthria. There was no wasting and tone was increased with spasticity of all four limbs and ankle clonus. There was mild weakness of finger extension and abduction and mild pyramidal weakness of both legs. All reflexes were brisk with bilateral extensor plantar responses. She had a broad based ataxic gait, fine finger movements were slow, with dysdiadochokinesis and a mild intention tremor; heel shin testing was inaccurate due to ataxia and spasticity.

Nerve conduction studies showed normal motor conduction but very reduced or absent sensory action potentials consistent with an axonal sensory neuropathy. Visual evoked responses were normal. Brain MRI was normal with no cerebellar atrophy. All CSF and blood investigations (including Vitamin $\mathrm{E}$ 
concentration) were normal. Her ECG showed prominent $\mathrm{R}$ waves from $\mathrm{V} 1$ to $\mathrm{V} 3$ with inverted $\mathrm{T}$ waves inferolaterally and a transthoracic echocardiogram disclosed moderate concentric left ventricular hypertrophy. Her condition has slowly deteriorated over four years of follow up, and she continues to have brisk reflexes and spasticity of her legs.

Patients 2 and 3

These were non-identical twins who both had FRDA and were the first cousins of the father of patient 1 . They were both seen at the Westminster Hospital in London in the 1950s from which the medical records containing these histories were obtained.

Patient 2 was normal until the age of 15 years, when progressive unsteadiness of walking, clumsiness of the hands, and slurred speech developed. She was thin with a mild thoracic kyphoscoliosis. Fundoscopy was normal. The remainder of the cranial nerves were normal. Her arms were hypotonic with mild wasting of the intrinsic hand muscles but normal power. There was pes cavus in the legs but no wasting, normal tone, and mild weakness bilaterally. She was areflexic with bilateral extensor plantar responses. Joint position and vibration sense were abnormal in the lower limbs but pain, temperature, and light touch were intact. There was pronounced gait and limb ataxia. Her ECG showed inverted $T$ waves in leads V1 to V5 and left ventricular hypertrophy. Examination of the CSF gave normal results. Her condition progressed and she became confined to a wheelchair and is known to have died in her late 20s.

Patient 3 was first seen in 1954 at the age of 21 after her sister's diagnosis. She was asymptomatic at initial assessment but on examination she had slight cerebellar incoordination affecting all four limbs and was areflexic with flexor plantar responses. She was tall and thin, with a pronounced thoracic kyphoscoliosis but no pes cavus. Extraocular movements and the rest of the examination were normal. These findings were thought to be consistent with early FRDA. Her condition progressed and she became confined to a wheelchair and died prematurely in her early 30 s.

\section{FAMILY B}

Patient 4

This patient was unstable when riding a bicycle at five years of age, and had great difficulty maintaining posture when horse riding two years later. He complained of back pain at the age of 9 and was found to have a mild thoracic scoliosis. An abnormal gait was noticed by his physical education teacher at secondary school with gradual progression throughout his teenage years, such that he required the use of a stick by the age of 20, and three years later required a wheelchair. Examination at the age of 17, disclosed slow tongue movement and a mild dysarthria. Ocular movements were dysmetric but fundoscopy and the remainder of the cranial nerves were normal. Tone was increased in the legs with normal power but there was moderate ataxia of all four limbs. Reflexes were preserved in the arms and legs with extensor plantar responses. His gait was broad based and very unsteady. Nerve conduction tests showed grossly reduced sensory nerve action potentials with normal conduction velocities. An ECG initially showed small $\mathrm{U}$ waves in the anterior chest leads but later showed widespread $\mathrm{T}$ wave abnormalities. $\mathrm{He}$ later developed paroxysmal supraventricular tachycardia which responded to disopyramide and later amiodarone. Transthoracic echocardiography showed concentric left ventricular hypertrophy initially although later he developed evidence of right ventricular overload. He died of cardiorespiratory failure at the age of 26.

\section{Patient 5}

At the age of 14 years, he noticed mild unsteadiness when running, and three years later found difficulty marching in a straight line. $\mathrm{He}$ became increasingly unsteady with clumsiness of the hands and mild slurring of speech. By the age of 34 he required a

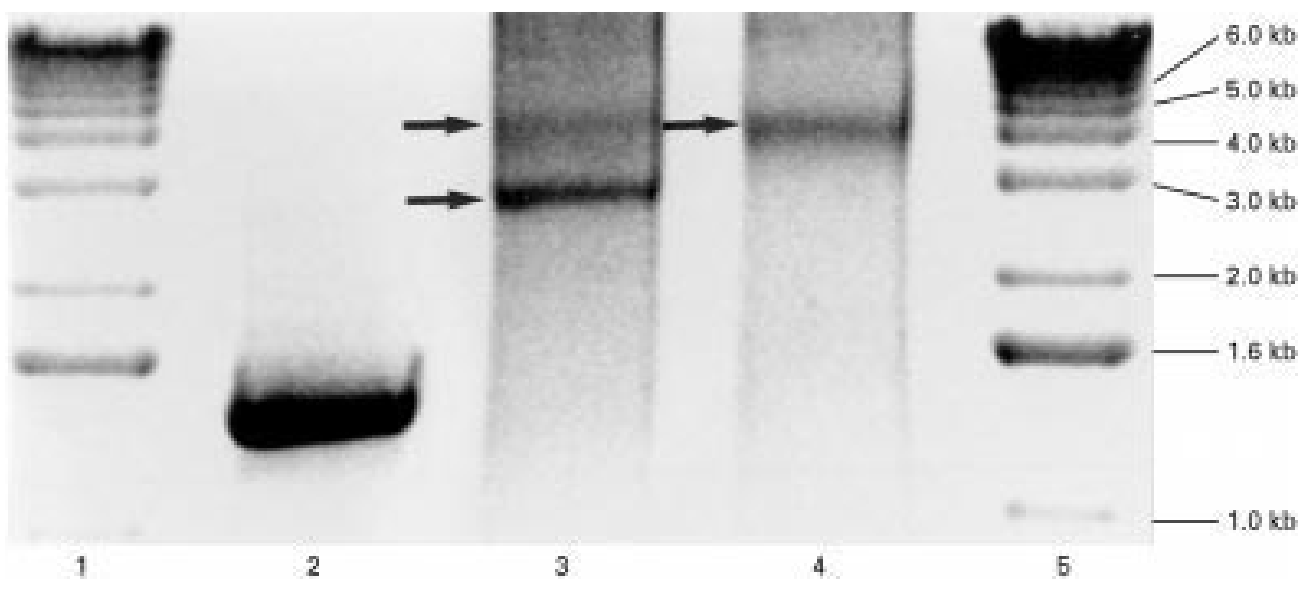

Electrophoretic strips labelled 1 to 5. Lanes 1 and 5 indicate kilobase (kb) ladders with bright bands at 1.0, 1.6, 2.0, 3.0, 4.0, 5.0, and $6.0 \mathrm{~kb}$. Lane 2 shows appearance in normal control with a band of approximately $1.4 \mathrm{~kb}$. The size of the repeat expansion is calculated by subtracting the length of the control band $(1.4 \mathrm{~kb})$ from the band size in the patient and dividing by a factor of three. Lane 3 shows patient 5 who is heterozygous for two repeat expansions of $3 \mathrm{~kb}$ and $4.5 \mathrm{~kb}$ (arrowed), equal to approximately 530 and 1000 trinucleotide repeats. Lane 4 shows patient 1 who is homozygous for a repeat expansion at $4 \mathrm{~kb}$ (arrowed), equal to about 870 trinucleotide repeats. 
wheelchair and his speech was more slurred. On examination, there was no optic atrophy but eye movements were abnormal with jerky pursuit and square wave jerks. He had cerebellar dysarthria. There was truncal ataxia and pronounced limb ataxia, mild pyramidal weakness of the legs, areflexia, and bilateral extensor plantar responses. Proprioception and vibration sense were impaired in the feet. There was no scoliosis or pes cavus. His CSF was normal as were vitamin $\mathrm{E}$ concentrations and white cell enzymes. Brain MRI showed no cerebellar or brainstem atrophy. Nerve conduction studies showed absent or very small sensory nerve action potentials consistent with an axonal sensory neuropathy. An ECG and transthoracic echocardiogram were normal.

There was no known family history of any neurodegenerative illnesses in patients 4 and 5 at the time of presentation. However, the mother of patient 4 has since been found to be related to patient 5 .

MOLECULAR GENETIC ANALYSIS

DNA was obtained from the surviving affected members of families A and B (patients 1 and 5) and analysed using a long range polymerase chain reaction with size separation on an agarose gel, as detailed in Lamont et al. ${ }^{13}$ Homozygous GAA trinucleotide repeat expansions of the FRDA gene were found in both patients (figure), estimated at about 870 repeats on each chromosome in patient 1 , and 530 and 1000 repeats in patient 5.

\section{Discussion}

For some years there has been uncertainty as to whether EOCA and FRDA might be related or distinct conditions. A few patients have been described with a phenotype very typical of FRDA including cardiomyopathy, but with preserved or even exaggerated reflexes, occurring either sporadically or in families in whom other affected members had the classic FRDA phenotype with areflexia. ${ }^{4589}$ Linkage studies of four families in which FRDA and EOCA phenotypes coexisted ${ }^{8}$ and three families with the EOCA phenotype alone ${ }^{8}$ showed linkage to the FRDA locus on chromosome 9q13-21.1, providing genetic evidence for a link between some patients with EOCA phenotype and FRDA. This has been taken a stage further with the discovery of a GAA trinucleotide repeat expansion in the $\mathrm{X} 25$ gene in patients with FRDA, ${ }^{10}$ and in patients such as patient 1 , who have phenotypic features of EOCA. ${ }^{11}{ }^{12}$ However the EOCA phenotype is genetically heterogeneous, ${ }^{7}$ and it is as yet unclear what proportion of cases carry a similar trinucleotide expansion in the X25 gene.

Before details of the neurological illness in their families were known, our patients 1 and 4 were diagnosed as having EOCA in view of their retained reflexes, which have been regarded as a major diagnostic criterion distinguishing EOCA from FRDA. ${ }^{1}$ Both patients we report on had cardiomyopathy, which is uncommon in patients with EOCA, with only one case $\mathrm{e}^{2}$ in 54 patients reported in three separate series. ${ }^{127}$ All previous cases with EOCA which mapped to the FRDA locus have had repolarisation abnormalities on ECG consistent with those seen in FRDA. ${ }^{78}$

Clinically, the diagnosis of FRDA in patients 2,3 , and 5 seems secure. The relation between these patients and patients 1 and 4 are consistent with autosomal recessive inheritance and are further examples of mixed phenotypes with and without retained reflexes in families with Friedreich's ataxia. Molecular genetic analysis confirms that the EOCA phenotype in patient 1 is caused by the same trinucleotide repeat expansion associated with a classic FRDA phenotype in family A. The detection of a FRDA mutation in patient 5 suggests that patient 4 who had retained reflexes, is also likely to have had FRDA. Such families illustrate that FRDA is unusually heterogeneous for an autosomal recessive disorder. Late onset FRDA, $^{14}{ }^{15}$ milder Acadian variants with a longer course of disease and older age at death, ${ }^{16}$ and variants with retained reflexes ${ }^{89}$ all result from trinucleotide repeats in the FRDA gene. ${ }^{11}{ }^{12}$

The normal X25 gene consists of eight to 22 trinucleotide repeats. Most patients with FRDA have two expanded alleles of different size, between 120 and 1700 repeats, with the mean number of 630 and 890 on the smaller and larger alleles respectively. The size of the abnormal expansion is responsible for some of the phenotypic variability, particularly age at onset and rate of disease progression, ${ }^{11} 12$ and some patients with clinical and electrophysiological EOCA have small GAA expansions. ${ }^{11}$ The size of the smallest expansion is the major determining factor accounting for $50 \%$ of the variability in age of onset. ${ }^{11}{ }^{12}$ The presence of cardiomyopathy also seems dependent on size of expansion, particularly that of the shorter allele, and is uncommon in patients with an expansion on the shorter allele of less than 700 repeats, ${ }^{11}$ possibly explaining why patient 5 in this report has not developed a cardiomyopathy. The genetic abnormality causing FRDA will allow further phenotypic characterisation; however, factors other than size of repeat expansion influence phenotypic variability of FRDA in its classic and "atypical" forms, limiting the predictive value of the test at the current time.

1 Harding AE. Early onset cerebellar ataxia with retained tendon reflexes: a clinical and genetic study of a disorder distinct from Friedreich's ataxia. $\mathcal{F}$ Neurol Neurosurg Psychiatry 1981;44:503-8.

2 Klockgether T, Petersen D, Grodd W, Dichgans J. Early onset cerebellar ataxia with retained reflexes: clinical electrophysiological and MRI observations in comparison with Friedreich's ataxia. Brain 1991;114:1559-73.

3 Harding AE. Friedreich's ataxia: a clinical and genetic study of 90 families with an analysis of early diagnostic criteria and intrafamilial clustering of clinical features. Brain 1981; 104:589-620.

4 Sherman I. Friedreich's disease. A report of two unusual cases. Arch Neurol Psychiatry 1934;32:1282-5.

5 Filla A, DeMichele G, Cavalcanti F, Santorelli F, Santoro L, Campanella G. Intrafamilial phenotype variation in Friedreich's disease: possible exceptions to diagnostic criteria. $\mathcal{F}$ Neurol 1991;238:147-50.

6 Chamberlain S, Shaw J, Rowland S, et al. Mapping of the mutation causing Friedreich's ataxia to human chromosome 9. Nature 1988;334:248-50.

7 Filla A, De Michele G, Cavalcanti F, et al. Clinical and genetic heterogeneity in early onset cerebellar ataxia with retained reflexes. $\mathcal{F}$ Neurol Neurosurg Psychiatry 1990;57: 667-70.

8 Palau F, DeMichele G, Vilchez JJ, et al. Early onset ataxia with cardiomyopathy and retained tendon reflexes maps to 
the Friedreich's ataxia locus on chromosome 9q. Ann Neurol 1995;37:359-62.

rol 1995;37:359-62. Klockgether T, Zuhlke C, Schultz JB, ataxia with retained reflexes: molecular genetics, clinical neurophysiology and magnetic resonance imaging. Neurology 1996;46:118-21.

10 Campuzzano V, Montermini L, Molto MD, et al. Friedreich's ataxia: autosomal recessive disease caused by an intronic GAA triplet repeat expansion. Science 1996;271 1423-7.

11 Durr A, Cossee M, Agid Y, et al. Clinical and genetic abnormalities in patients with Friedreich's ataxia. $N$ Engl f Med 1996;335:1169-75.

12 Filla A, DeMichele G, Cavalcanti F, et al. The relationship between trinucleotide (GAA) repeat length and clinical features in Friedreich's ataxia. Am F Hum Genet 1996;59: 554-60.
13 Lamont PJ, Davis MB, Wood NW. Identification and sizing of the GAA trinucleotide repeat expansion of Friedreich's ataxia in 55 patients; clinical and genetic correlates. Brain 1997;120:673-80.

14 Klockgether T, Chamberlain S, Wullner U, et al. Late onset Friedreich's ataxia: molecular genetics, clinical neurophysiology, and magnetic resonance imaging. Arch Neurol 1993; 50:803-6.

15 DeMichele G, Filla A, Cavalcanti F, et al. Late onset Friedreich's disease: clinical features and mapping of mutation to FRDA locus. F Neurol Neurosurg Psychiatry 1994;57: 977-9.

16 Keats JB, Ward LJ, Shaw J, Wickrenmasinghe A, Chamberlain S. "Acadian" and "classical" forms of Friedreich ataxia are probably caused by mutations at the same locus. $A m \mathcal{F}$ Hum Genet 1989;33:266-8. 